\title{
Features of Anti-radical Activity and Antioxidant Sperm Status in Men Living in the Western Urals
}

\author{
O.V. Dolgikh ${ }^{1, *}$ and D.G. Dianova ${ }^{2}$ \\ ${ }^{1}$ Department of Immunobiological Diagnostic Methods, Federal Scientific Center for Medical and Preventive \\ Technologies for Managing Public Health Risks, Perm, Russia \\ ${ }^{2}$ Federal Scientific Center for Medical and Preventive Technologies for Managing Public Health Risks, Perm, Russia \\ *Corresponding author. Email: oleg@fcrisk.ru
}

\begin{abstract}
Inflammatory diseases in the urogenital tract of men living in different climatic conditions often cause infertility. The study aims to assess the antioxidant status and antiradical activity of sperm in men with chronic prostatitis living in the Western Urals. 55 men aged 23 to 38 years with inflammatory diseases of the prostate gland living in the Western Urals were examined. Group 1 consisted of 25 men with chronic prostatitis in remission, group 2 was composed of 30 people with chronic prostatitis at the acute stage. The level of total antiradical activity of sperm (TAAS) was studied using the SF PE-5300 (Russia), the activity of superoxide dismutase (SOD) was studied on the enzyme immunoassay analyzer Sunrise (Tecan, Austria). Results are presented as arithmetic mean (M), standard error (m) and $95 \%$ confidence interval for mean $(95 \% \mathrm{CI})$. To test the null hypotheses about the equality of mean values between two independent groups with a normal distribution, the two-sample Student's test was used. The significance level at which the null hypotheses were tested was 0.05 . According to the assessment of the antioxidant status of sperm, there was a statistically significant $(\mathrm{p}=0.039) 20 \%$ increase in the total antiradical activity of sperm in patients with an acute inflammatory process in the prostate gland compared to the similar indicator identified in men without symptoms of the acute inflammation. In the group of patients with acute chronic prostatitis, the share of samples with a significant increase in the level of total antiradical activity of seminal plasma compared to the reference level was $33.3 \%$ versus $23.3 \%$ in men with chronic prostatitis during the remission; the frequency of excess was 1.5 times. The evaluation of sperm parameters showed that the activity of superoxide dismutase in both groups was within the reference range. In the seminal plasma of men with chronic prostatitis during an exacerbation, in $8.8 \%$ of samples, the activity of superoxide dismutase was lower, and in patients with the chronic process in the prostate gland during the remission, the activity of superoxide dismutase was below the reference level in $3.3 \%$ of the samples, the frequency of a decrease was 2.7. Thus, with chronic prostatitis, the imbalance in the oxidation-antioxidation system plays a key role in the development of impaired spermatogenesis, and markers of redox processes can serve as informative indicators of the
\end{abstract}

Keywords: anti-radical activity, antioxidant sperm status, urogenital tract, inflammatory diseases, Western Urals, climatic conditions

\section{INTRODUCTION}

It is known that reactive oxygen species (ROS) play the most significant role in the regulation of the vital activity of the organism and the functional activity of the cell in various climatic and geographical conditions $[1,2]$. Of particular relevance is an assessment of the universal protective and adaptive reactions of the multicellular organism to mechanical, physical, chemical, biological and other environmental factors in order to localize and / or eliminate damaging agents, as well as to restore (or replace) damaged tissues [3]. Researchers argue about the participation of reactive oxygen species (ROS), generated in the inflammation 
focus, in redox-regulated signalling cascades in the target cells of the body. Physiological or pathological effects of the ROS are realized depending on the factor influencing the cell, time and intensity of the factor. The result of ROS-mediated signalling is a change in the level of gene expression, activation of nuclear transcription factors, initiation or inhibition of apoptosis. It has been proven that ROS overproduction can damage cells and tissues and contribute to the chronic inflammation underlying metabolic and neurodegenerative disorders, cardiovascular pathology, and respiratory system diseases in men in various climatic conditions [1-4].

The main sources of reactive oxygen species in semen are leukocytes and sperm cells. It is the ROS level that regulates the spermatogenesis [4]. The participation of reactive oxygen species in the condensation of chromatin in spermatozoa has been proven, and the role of ROS in the regulation of proliferation and apoptosis of spermatogonia has been established [5]. Various intracellular and extracellular stimuli can provoke excessive accumulation of reactive oxygen species and lead to detrimental effects on sperm function, in particular, damage to sperm DNA, which reduces the likelihood of pregnancy [6]. The antioxidant system consisting of enzymatic and non-enzymatic links protects the cell from the negative consequences of ROS. It is relevant to assess the antioxidant status of sperm plasma in men with inflammatory processes in the prostate gland, living in different climatic conditions.

The aim is to evaluate the antioxidant status and antiradical activity of sperm in men with chronic prostatitis living in the Western Urals.

\section{MATERIALS AND METHODS}

The study was conducted in compliance with the ethical requirements fixed in the 2000 Helsinki Declaration by the WMA and the Protocol of the Council of Europe Convention on Human Rights and Biomedicine (1999). In total, 55 men aged 23 to 38 years with inflammatory diseases of the prostate gland living in the Western Urals were examined. Group 1 consisted of 25 people with chronic prostatitis in remission, group 2 - of 30 men with acute chronic prostatitis.

The level of total antiradical activity of sperm (TAAS) was studied using the SF PE-5300 (Russia); the activity of superoxide dismutase (SOD) was studied with the enzyme immunoassay analyzer Sunrise (Tecan, Austria)

The distribution of quantitative data was checked using the Kolmogorov-Smirnov statistical test. The arithmetic mean (M), standard error $(\mathrm{m})$, and $95 \%$ confidence interval for the mean $(95 \% \mathrm{CI})$ were used to describe data that were normally distributed. To test the null hypotheses about the equality of mean values between two independent groups with a normal distribution, the two-sample Student's t-test was used. The significance level at which the null hypotheses were tested was 0.05. Statistical analysis of the data was carried out in Statistica 6.0 (StatSoft, USA)

\section{RESULTS}

It was found that the average value of the total antiradical activity of sperm plasma in the group of men with chronic prostatitis without exacerbation corresponded to the norm (Table 1). The study of sperm quality revealed a statistically significant $(p=0.039)$ 1.2-fold increase in the TAAAS level in men with acute chronic prostatitis relative to the values obtained in men without symptoms of the acute inflammatory process. Evaluation of sperm parameters showed that the activity of superoxide dismutase in both groups was within the reference values.

The TAAS of seminal plasma in group 2 (exacerbation stage) varied from 207 to $3010 \mu \mathrm{M} / \mathrm{L}$, and in group 1 (without exacerbation) it varied from 40 to $1570 \mu \mathrm{M} / \mathrm{L}$ (Table 2). In men with an acute inflammatory process (group 2), the share of samples with a significant increase in the level of total antiradical activity of seminal plasma and activity of superoxide dismutase was 33.3 and 28.8 versus 23.3 and 16.6 in men with chronic prostatitis during the remission (group 1); the frequency of an excess was 1.6 times.

Table 1. Indicators of antioxidant activity of spermoplasma in men, m (m); $95 \%$ CI

\begin{tabular}{|l|l|l|l|l|l|}
\hline Indicator & Reference level & Group $1 n=25$ & Group 2 $n=30$ & $t$ & $p$ \\
\hline \multirow{2}{*}{ TAAS, $\mu \mathrm{M} / \mathrm{L}$} & $500-1000$ & $\begin{array}{l}794.88(47.40) \\
755.68-834.08\end{array}$ & $\begin{array}{l}960.69(62.92) \\
889.39-1032.53\end{array}$ & 2.11 & 0.039 \\
\hline \multirow{2}{*}{ SOD, U/ml } & $164-270$ & $245.34(56.25)$ & $242.41(65.93)$ & 0.030 & 0.973 \\
& & $224.34-266.35$ & $222.60-262.21$ & \\
\hline
\end{tabular}

The share of sperm plasma samples with a reduced level of TAAS in group 2 (exacerbation stage) was
$17.7 \%$ versus $26.6 \%$ in group 1 (without exacerbation), the frequency of a decrease was 1.5 times. In the 
seminal plasma of men with chronic prostatitis during the exacerbation (group 2) in $8.8 \%$ of the samples, the SOD activity was below the standard values, while in $3.3 \%$ of the samples of men with a chronic process in the prostate gland during the remission (group 1), the superoxide dismutase activity was below the reference value; the frequency of a reduction was 2.7 times.

Table 2. The frequency of registration of samples of antioxidant status indicators with deviations from reference values, $\%$

\begin{tabular}{|c|c|c|c|c|c|c|}
\hline \multirow{3}{*}{ Indicator } & \multicolumn{3}{|l|}{ Group 1 n=25 } & \multicolumn{3}{|l|}{ Group 2 n=30 } \\
\hline & \multirow{2}{*}{ Range of values } & \multicolumn{2}{|c|}{ Abnormal samples } & \multirow{2}{*}{ Range of values } & \multicolumn{2}{|c|}{ Abnormal samples } \\
\hline & & higher & lower & & higher & lower \\
\hline TAAS, $\mu \mathrm{M} / \mathrm{L}$ & 40-1570 & 23.3 & 26.6 & 207-3010 & 33.3 & 17.7 \\
\hline $\mathrm{SOD}, \mathrm{U} / \mathrm{ml}$ & $154.21-398.54$ & 16.6 & 3.3 & $114.85-444.43$ & 28.8 & 8.8 \\
\hline
\end{tabular}

An increase in the total antiradical activity of sperm plasma during the active inflammatory process suggests an excessive generation of reactive oxygen species against the background of the oxidative stress in sperm. Under the physiological conditions, various nonenzymatic and enzymatic systems are responsible for redox homeostasis; however, the imbalance between the prooxidant and antioxidant systems during inflammation causes metabolic disorders and energy supply of germ cells. Obviously, oxidative stress in sperm can cause male infertility.

It has been proven that an increased level of reactive oxygen species in inflammatory diseases of the urogenital tract is due to the unsatisfactory sperm profile, characterized by a high share of inactive mitochondria, abnormal expression of mitochondrial proteins, and changes in the calcium signaling cascade [7-10]. The altered level of calcium in the sperm was due to the low activity of $\mathrm{Na}+$.- $\mathrm{K}+$-ATPase of the sperm, which reduces the mobility of the male reproductive cell [11]. Changes in the functional activity of mitochondria can cause impaired sperm motility [12]. The participation of ROS in the dephosphorylation of glycogen synthase kinase $3 \alpha$ (GSK), a negative regulator of sperm motility, has been suggested [13]. The significant effect of glycogen synthase- $3 \beta$ kinase on the opening / closing of the mitochondrial pore has been proven [1-4].

We should note that reactive oxygen species induce multiple cellular physiological processes (proliferation, differentiation, apoptosis, etc.) in physiological conditions $[14,15]$. It is interesting that sperm cells with defective morphology turn out to generate reactive oxygen species in greater amounts, for example, as a response to inflammation or negative exposure to exogenous factors, than sperm cells with proper structure. In case there is acute inflammation, ROS are generated in significant quantities and it results, in particular, in Nrf2 / ARE activation and induction of some enzymes [16]. Nrf2 / ARE activation increases activity of superoxide dismutase and glutathione peroxidase (antioxidants), certain enzymes in P-450 cytochrome family (enzymes participating in I detoxification phase), glutathione transferase (enzymes participating in II detoxification phase) [17]. ROS $[18,19]$ and glutathione [20] are assumed to participate in a chain for transferring signals that activated SOD and expression of its genes at transcription level. Superoxide dismutase deficiency in a body results in weaker protective functions and damage to cells done by free radicals. Meanwhile increased SOD activity is able to induce excessive $\mathrm{H}_{2} \mathrm{O}_{2}$ quantities in a body and, consequently, result in toxic damage to cells [21].

Lipid peroxidation that occurs mostly due to effects produced by reactive oxygen species makes for changes in expression of surface membrane receptors and also can induce disorders in activity of matrix enzymes in mitochondria and dysfunction of components in the respiratory chain. It has been shown on many cellular models that increased ROS generation can induce cell death and internal bio-energetic reserve is a basic factor that determines cellular response; it has also been shown that a cell should be provided with sufficient energy for starting detoxification and apoptosis. In case there is irreversible mitochondria dysfunction and energy crisis, "string lethal" stimuli induce cellular necrosis. Obviously, oxidative stress induces transcription of specific genes, activates the most significant enzymes, modulates intracellular signal pathways that coordinate cell life cycle; given all that it can modify the most significant functions performed by gamete changing its vital activity depending on duration and extent of oxidation-recovery imbalance [22-25].

Quantitative and qualitative properties of human sperm tend to deteriorate and it has been detected worldwide recently together with growing number if dysfunctions in the reproductive system; it is a serious medical problem that seems only to aggravate thus overstepping medical boundaries and becoming a social and demographic issue. Given that, it is truly vital to examine reasons for male infertility including studies performed on men living in different climatic and 
geographic conditions. According to some authors, oxidative stress which causes male infertility in 30-80 $\%$ cases [26] also plays a key role in DNA fragmentation [27], lower sperm cells mobility [28], and male gamete death [29]. Reproductive health is a significant component in men's overall health and welfare. Improving and preserving reproductive potential allow avoiding sexual diseases and provide efficient preservation and growth of male fertility.

\section{RESULTS}

Thus, the analysis of the antioxidant status of sperm plasma in men with chronic prostatitis living in the Western Urals determined its specific features taking into account the inflammatory process. It was found that in men with chronic prostatitis at the decompensation stage, a statistically significant $(\mathrm{p}=0.039)$ 1.2-fold decrease in the TAAS level of sperm was observed

The results indicate a high potential antiradical activity of sperm plasma in men with inflammatory diseases, which may indicate an excessive increase in the production of free radicals during the exacerbation. The TAAS changes in sperm plasma in men with chronic diseases of the urogenital tract play a pathogenetic role in the development of spermatogenesis disorders, and markers of redox processes can serve as informative indicators of the inflammatory process in men living in the Western Urals.

\section{REFERENCES}

[1] M.A. Chelombitko, Role of reactive oxygen species in inflammation: A minireview, Moscow Univ. Biol. Sci. Bull. 73 (2018) 199-202.

[2] O.V. Dolgikh, D.G. Dianova, O.A. Kazakova, Vanadium in the environment as a risk factor causing negative modification of cell death (scientific review), Health Risk Anal. 4 (2021) 156-169.

[3] I.D. Magamedov, L.P. Pivovarova, O.B. Ariskina et al., Markers of inflammation and oxidative stress in the treatment of acute ischemia of the lower limbs, Rus. J. of Immunol. 22(2-2) (2019) 10541056.

[4] G. Lavranos, M. Balla, A. Tzortzopoulou et al., Investigating ROS sources in male infertility: A common end for numerous pathways, Reprod. Toxicol. 34(3) (2012) 298-307.

[5] Sj. Chen, J.P. Allam, Yg. Duan, Influence of reactive oxygen species on human sperm functions and fertilizing capacity including therapeutical approaches, Arch. Gynecol. Obstet. 88 (2013) 191199.
[6] P. Gharagozloo, R. Aitken, The role of sperm oxidative stress in male infertility and the significance of oral antioxidant therapy, Human. Reprod. 26(7) (2011) 1628-1640.

[7] R. Dcunha, R.S. Hussein, H. Ananda et al., Current insights and latest updates in sperm motility and associated applications in assisted reproduction, Reprod. Sci. 2020. Retrieved from: https://doi.org/10.1007/s43032-020-00408-y

[8] A. Agarwal, R. Sharma, A. Harlev, S. Esteves, Effect of varicocele on semen characteristics according to the new 2010 World Health Organization criteria: a systematic review and meta-analysis, Asian. J. Andrology 18(2) (2016) 163-170.

[9] L. Samanta, A. Agarwal, N. Swain et al., Proteomic signatures of sperm mitochondria in varicocele: clinical use as biomarkers of varicocele associated infertility, J. Urol. 200(2) (2018) 414 422.

[10] C.G. Blumer, R.M. Fariello, A.E. Restelli et al., Sperm nuclear DNA fragmentation and mitochondrial activity in men with varicocele, Fertil. Steril. 90(5) (2008) 1716-1722.

[11] S.A. Banihani, F.H. Khasawneh, Effect of lansoprazole on human sperm motility, sperm viability, seminal nitric oxide production, and seminal calcium chelation, Res. Pharm. Sci. 13(5) (2018) 460-468.

[12] S. Baklouti-Gargouri, M. Ghorbel, A.B. Mahmoud et al., Identification of a novel m. 9588G> a missense mutation in the mitochondrial COIII gene in asthenozoospermic Tunisian infertile men, J. Assist. Reprod. Genet. 31(5) (2014) 595-600.

[13] M. Kanter, C. Aktas, M. Erboga, Heat stress decreases testicular germ cell proliferation and increases apoptosis in short term: an immunohistochemical and ultrastructural study, ToxicolInd. Health 29(2) (2013) 99-113.

[14] I.D. Magamedov, L.P. Pivovarova, O.B. Ariskina et al., Markers of inflammation and oxidative stress in treatment of acute lower extremities ischemia, Rus. Immunol. J. 22(2-2) (2019) 1054-1056.

[15] G. Lavranos, M. Balla, A. Tzortzopoulou et al., Investigating ROS sources in male infertility: A common end for numerous pathways, Reprod. Toxicol. 34(3) (2012) 298-307.

[16] W. Li, C. Busu, M.L. Circu et al., Glutathione in cerebral microvascular endothelial biology and pathobiology: implications for brain homeostasis, Int. J. Cell. Biol. 14 (2012) 1002-1012. 
[17] N.K. Zenkov, E.B. Men'shchikova, V.O. Tkachyov, Certain principles and mechanisms of redox regulation, Oxygen and antioxid. 1 (2009) 3-64.

[18] M.B. Hampton, S. Orrenius, Dual regulation of caspase activity by hydrogen peroxide: implications for apoptosis, FEBS Lett. 414 (1997) 552-556.

[19] S. Herbette, C. Lene, D. de Iabrouhe et al., Transcripts of sunflower antioxidant scavengers of the SOD and GPX families accumulate differentially in response to downy mildew infection, photohormones, reactive oxygen species, nitric oxide, protein kinase and phosphatase inhibitors, Physiol. Plant. 119 (2003) 418-428.

[20] N. Estévez-Calvar, A. Romero, A. Figueras et al., Genes of the mitochondrial apoptotic pathway in Mytilus galloprovincialis, PLoS One 8(4) (2013) 61502.

[21] B. Long, T. Gan, R.C. Zhang et al., miR-23a regulates cardiomyocyte apoptosis by targeting manganese superoxide dismutase, Mol. Cells. 40(8) (2017) 542-549.

[22] M.A. Chelombitko, Role of reactive oxygen species in inflammation: A minireview, Moscow Univ. Biol. Sci. Bull. 73 (2018) 199-202.

[23] Sj. Chen, J.P. Allam, Yg. Duan, Influence of reactive oxygen species on human sperm functions and fertilizing capacity including therapeutical approaches, Arch. Gynecol. Obstet. 288 (2013) 191-199.

[24] P. Gharagozloo, R. Aitken, The role of sperm oxidative stress in male infertility and the significance of oral antioxidant therapy, Human. Reprod. 26(7) (2011) 1628-1640.

[25] G. Lavranos, M. Balla, A. Tzortzopoulou et al., Investigating ROS sources in male infertility: A common end for numerous pathways, Reprod. Toxicol. 34(3) (2012) 298-307.

[26] A. Agarwal, M. Arafa, H. Okada et al., Multicenter evaluation of oxidation-reduction potential by the MiOXSYS in males with abnormal semen, Asian. J. Androl. 21(6) (2019) 565-569.

[27] S.R. Salian, G. Nayak, S. Kumari et al., Supplementation of biotin to sperm preparation medium enhances fertilizing ability of spermatozoa and improves preimplantation embryo development, J. Assist. Reprod. Genet. 36 (2019) 255-266.
[28] V.V. Evdokimov, O.B. Zhukov, Yu.V. Kastrikin et al., Oxidative stress and pathozoospermia, Experim. and clin. Urol. 2 (2017) 73-77.

[29] Y. Chen, J. Yang, Y. Wang et al., Zinc deficiency promotes testicular cell apoptosis in mice, Biol. Trace. Elem. Res. 195 (2020) 142-149.

[30] [9] A. Pnueli, In transition from global to modular temporal reasoning about programs, in: K.R. Apt (Ed.), Logics and Models of Concurrent Systems, Springer, Berlin, Heidelberg, 1984, pp. 123-144. DOI: https://doi.org/10.1007/978-3-642-82453-1_5

[31] B. Meyer, Applying "Design by Contract", Computer 25(10) (1992) 40-51. DOI: https://doi.org/10.1109/2.161279

[32] S. Bensalem, M. Bogza, A. Legay, T.H. Nguyen, J. Sifakis, R. Yan, Incremental component-based construction and verification using invariants, in: Proceedings of the Conference on Formal Methods in Computer Aided Design (FMCAD), IEEE Press, Piscataway, NJ, 2010, pp. 257-256.

[33] H. Barringer, C.S. Pasareanu, D. Giannakopolou, Proof rules for automated compositional verification through learning, in Proc. of the 2nd International Workshop on Specification and Verification of Component Based Systems, 2003.

[34] M.G. Bobaru, C.S. Pasareanu, D. Giannakopoulou, Automated assume-guarantee reasoning by abstraction refinement, in: A. Gupta, S. Malik (Eds.), Proceedings of the Computer Aided Verification, Springer, Berlin, Heidelberg, 2008, pp. 135-148. DOI: https://doi.org/10.1007/978-3540-70545-1_14 